$\operatorname{SINP} / \mathrm{TNP} / 01-22$

\title{
Three Generation Neutrino Oscillation Parameters after SNO
}

\author{
Abhijit Bandyopadhyay!, Sandhya Choubey [2, Srubabati Goswami [], \\ Kamales Kar ${ }^{\text {f }}$ \\ Saha Institute of Nuclear Physics, \\ 1/AF, Bidhannagar, Kolkata 700 064, INDIA
}

\begin{abstract}
We examine the solar neutrino problem in the context of the realistic three neutrino mixing scenario including the SNO charged current $(\mathrm{CC})$ rate. The two independent mass squared differences $\Delta m_{21}^{2}$ and $\Delta m_{31}^{2} \approx \Delta m_{32}^{2}$ are taken to be in the solar and atmospheric ranges respectively. We incorporate the constraints on $\Delta \mathrm{m}_{31}^{2}$ as obtained by the SuperKamiokande atmospheric neutrino data and determine the allowed values of $\Delta m_{21}^{2}, \theta_{12}$ and $\theta_{13}$ from a combined analysis of solar and $\mathrm{CHOOZ}$ data. Our aim is to probe the changes in the values of the mass and mixing parameters with the inclusion of the SNO data as well as the changes in the two-generation parameter region obtained from the solar neutrino analysis with the inclusion of the third generation. We find that the inclusion of the SNO CC rate in the combined solar + CHOOZ analysis puts a more restrictive bound on $\theta_{13}$. Since the allowed values of $\theta_{13}$ are constrained to very small values by the $\mathrm{CHOOZ}$ experiment there is no qualitative change over the two generation allowed regions in the $\Delta m_{21}^{2}-\tan ^{2} \theta_{12}$ plane. The best-fit comes in the LMA region and no allowed area is obtained in the SMA region at $3 \sigma$ level from combined solar and $\mathrm{CHOOZ}$ analysis.
\end{abstract}

PACS numbers(s): 14.60.Pq, 12.15.Ff, 26.65.+t.

\footnotetext{
1abhi@theory.saha.ernet.in

${ }^{2}$ sandhya@theory.saha.ernet.in

${ }^{3}$ sruba@theory.saha.ernet.in

${ }^{4}$ kamales@theory.saha.ernet.in
} 


\section{Introduction}

The recent results on charged current measurement from Sudbury Neutrino Observatory (SNO) [1] have confirmed the solar neutrino shortfall as observed in the earlier experiments [2, 3, 4, 5]. A comparison of the SuperKamiokande and SNO results establishes the presence of non-electron flavor component in the solar neutrino flux received at earth (at more than $3 \sigma$ level) in a model independent manner [1, 6, 7, 8]. Neutrino oscillation provides the most popular explanation to this anomaly. Two generation analysis of the solar neutrino data including the SNO results has been performed by various groups [0, 9, 10, 11, 12, 13, 14, 15]. All these analyses agree that the best description to the data on the total rates and the day/night spectrum data of the SuperKamiokande (SK) collaboration is provided by the Large Mixing Angle (LMA) MSW solution $\left(\Delta m_{\odot}^{2} \sim 10^{-5} \mathrm{eV}^{2}\right)$, though the low $\Delta \mathrm{m}_{\odot}^{2}$ solution (LOW-QVO) $\left(\Delta m_{\odot}^{2} \sim 10^{-9}-10^{-7} \mathrm{eV}^{2}\right)$ and the vacuum oscillation (VO) solutions $\left(\Delta m^{2} \sim 4.5 \times 10^{-10} \mathrm{eV}^{2}\right)$ are also allowed. The Small Mixing Angle (SMA) MSW solution is largely disfavoured with no allowed contour in the mass-mixing plane at the $3 \sigma$ level f . On the other hand, for the explanation of the atmospheric neutrino anomaly the two generation oscillation analysis of the atmospheric neutrino data requires $\Delta \mathrm{m}_{\text {atm }}^{2} \sim 10^{-3} \mathrm{eV}^{2}$ [16]. Since the allowed ranges of $\Delta \mathrm{m}_{\odot}^{2}$ and $\Delta \mathrm{m}_{\text {atm }}^{2}$ are completely non-overlapping, to explain the solar and atmospheric neutrino data simultaneously by neutrino oscillation, one requires at least two independent mass-squared differences and consequently three active neutrino flavors which fits very nicely with the fact that to date we have observed three neutrino flavours in nature. Thus to get the complete picture of neutrino masses and mixing a three generation analysis is called for. Apart from the solar and atmospheric neutrinos positive evidence for neutrino oscillation is also published by the LSND experiment [17] and although there had been several attempts to explain all the three evidences in a three generation picture it is now widely believed that to accommodate the LSND results one has to introduce an additional sterile neutrino [18, 19]. For the purpose of this analysis we ignore the LSND results. We incorporate the negative results from the $\mathrm{CHOOZ}$ reactor experiment on the measurement of $\overline{\nu_{e}}$ oscillation by disappearance technique [20]. CHOOZ is sensitive to $\Delta \mathrm{m}_{\mathrm{CHOOZ}}^{2} \gtrsim 10^{-3}$ $\mathrm{eV}^{2}$ which is the range probed in the atmospheric neutrino measurements and together they can put important constraints on the three neutrino mixing parameters. We consider the three flavour picture with

- $\Delta m_{21}^{2}=\Delta m_{\odot}^{2}, \bullet \Delta m_{31}^{2}=\Delta m_{C H O O Z}^{2} \simeq \Delta m_{\text {atm }}^{2}=\Delta m_{32}^{2}$.

\footnotetext{
${ }^{5}$ Only exception is the analysis of [9] which get a small allowed region for the SMA solution due to a slight difference in the treatment of the data.
} 
Three flavor oscillation analysis of solar, atmospheric and CHOOZ data assuming this mass spectrum was performed in pre SNO era by different groups [21, 22, 23]. We investigate the impact of the charged current measurement at SNO on neutrino mass and mixing in a three flavor scenario and present the most up to date status of the allowed values of three flavor oscillation parameters.

The plan of the paper is as follows. In section 1 we present the relevant probabilities. In section 3 we discuss the $\chi^{2}$-analysis method and the results. We end in section 4 with some discussion and conclusions.

\section{Calculation of Probabilities}

The three-generation mixing matrix that we use is

$$
\begin{aligned}
U & =R_{23} R_{13} R_{12} \\
& =\left(\begin{array}{ccc}
c_{13} c_{12} & s_{12} c_{13} & s_{13} \\
-s_{12} c_{23}-s_{23} s_{13} c_{12} & c_{23} c_{12}-s_{23} s_{13} s_{12} & s_{23} c_{13} \\
s_{23} s_{12}-s_{13} c_{23} c_{12} & -s_{23} c_{12}-s_{13} s_{12} c_{23} & c_{23} c_{13}
\end{array}\right)
\end{aligned}
$$

where we neglect the $\mathrm{CP}$ violation phases. This is justified as one can show that the survival probabilities $P_{e e}$ of the electron neutrinos do not depend on these phases. The above choice has the advantage that the matrix elements $\mathrm{U}_{e 1}$, $\mathrm{U}_{e 2}$ and $\mathrm{U}_{e 3}$ relevant for the solar neutrino problem becomes independent of $\theta_{23}$ while the elements $\mathrm{U}_{e 3}, \mathrm{U}_{\mu 3}$ and $\mathrm{U}_{\tau 3}$ relevant for the atmospheric neutrino problem are independent of $\theta_{12}$. The mixing angle common to both solar and atmospheric neutrino sectors is $\theta_{13}$ which, as we will see, is constrained severely by the $\mathrm{CHOOZ}$ data.

\subsection{Solar Neutrinos}

The general expression for the survival amplitude for an electron neutrino arriving on the earth from the sun, in presence of three neutrino flavours is given by 24

$$
A_{e e}=A_{e 1}^{\odot} A_{11}^{v a c} A_{1 e}^{\oplus}+A_{e 2}^{\odot} A_{22}^{v a c} A_{2 e}^{\oplus}+A_{e 3}^{\odot} A_{33}^{v a c} A_{3 e}^{\oplus}
$$

where $A_{e k}^{\odot}$ gives the probability amplitude of $\nu_{e} \rightarrow \nu_{k}$ transition at the solar surface, $A_{k k}^{v a c}$ gives the transition amplitude from the solar surface to the earth surface, $A_{k e}^{\oplus}$ denotes the $\nu_{k} \rightarrow \nu_{e}$ transition amplitudes inside the earth. One can write the transition amplitudes in the sun as an amplitude part times a phase part

$$
A_{e k}^{\odot}=a_{e k}^{\odot} e^{-i \phi_{k}^{\odot}}
$$


$a_{e k}^{\odot^{2}}$ can be expressed as

$$
a_{e k}^{\odot}=\sum_{j=1,2,3} X_{k j} U_{j e}^{\odot^{2}}
$$

where $X_{k j}$ denotes the non-adiabatic jump probability between the $\mathrm{j}^{\text {th }}$ and $\mathrm{k}^{\text {th }}$ state and $U_{j e}^{\odot}$ denotes the mixing matrix element between the flavour state $\nu_{e}$ and the mass state $\nu_{j}$ in sun. $A_{k k}^{v a c}$ is given by

$$
A_{k k}^{v a c}=e^{-i E_{k}\left(L-R_{\odot}\right)}
$$

where $E_{k}$ is the energy of the state $\nu_{k}, L$ is the distance between the center of the Sun and Earth and $R_{\odot}$ is the solar radius. For a two slab model of the earth - a mantle and core with constant densities of 4.5 and $11.5 \mathrm{gm} \mathrm{cm}^{-3}$ respectively, the expression for $A_{k e}^{\oplus}$ can be written as (assuming the flavor states to be continuous across the boundaries) [25],

$$
A_{k e}^{\oplus}=\sum_{\substack{i, j, l, \alpha, \beta, \sigma}} U_{e l}^{M} e^{-i \psi_{l}^{M}} U_{\alpha l}^{M} U_{\alpha i}^{C} e^{-i \psi_{i}^{C}} U_{\beta i}^{C} U_{\beta j}^{M} e^{-i \psi_{j}^{M}} U_{\sigma j}^{M} U_{\sigma k}
$$

where $(i, j, l)$ denotes mass eigenstates and $(\alpha, \beta, \sigma)$ denotes flavor eigenstates, $U^{M}$ and $U^{C}$ are the mixing matrices in the mantle and the core respectively and $\psi^{M}$ and $\psi^{C}$ are the corresponding phases picked up by the neutrinos as they travel in the mantle and the core of the Earth.

$$
\begin{aligned}
P_{e e} & =\left|A_{e e}\right|^{2} \\
& =\Sigma_{k} a_{e i}^{\odot}\left|A_{k e}^{\oplus}\right|^{2}+\sum_{l>k} 2 a_{e k}^{\odot} a_{e l}^{\odot} R e\left[A_{k e}^{\oplus} A_{l e}^{\oplus} e^{i\left(E_{l}-E_{k}\right)\left(L-R_{\odot}\right)} e^{i\left(\phi_{l}^{\odot}-\phi_{k}^{\odot}\right)}\right]
\end{aligned}
$$

This is the most general expression for the probability [26]. Since for our case $\Delta_{31} \approx \Delta_{32}$ is $\sim 10^{-3} \mathrm{eV}^{2}$ the phase terms $e^{i\left(E_{3}-E_{1}\right)\left(L-R_{\odot}\right)}$ and $e^{i\left(E_{3}-E_{2}\right)\left(L-R_{\odot}\right)}$ average out to zero. Therefore the probability simplifies to

$$
\begin{aligned}
P_{e e}= & a_{e 1}^{\odot 2}\left|A_{1 e}^{\oplus}\right|^{2}+a_{e 2}^{\odot 2}\left|A_{2 e}^{\oplus}\right|^{2}+a_{e 3}^{\odot 2}\left|A_{3 e}^{\oplus}\right|^{2} \\
& +2 a_{e 1}^{\odot} a_{e 2}^{\odot} R e\left[A_{1 e}^{\oplus} A_{2 e}^{\oplus *} e^{i\left(E_{2}-E_{1}\right)\left(L-R_{\odot}\right)} e^{i\left(\phi_{2}^{\odot}-\phi_{1}^{\odot}\right)}\right]
\end{aligned}
$$

The mixing matrix elements in matter are different from those in vacuum and it is in general a difficult task to find the matter mixing angles and eigenvalues for a $3 \times 3$ matrix. However in our case since $\Delta \mathrm{m}_{31}^{2}>>\Delta \mathrm{m}_{21}^{2} \approx$ the matter potential in sun, the $\nu_{3}$ state experiences almost no matter effect and MSW resonance can occur between $\nu_{2}$ and $\nu_{1}$ states. Under this approximation the three generation survival probability for the electron neutrino can be expressed as,

$$
P_{e e}=c_{13}^{4} P_{e e}^{2 g e n}+s_{13}^{4}
$$


where $P_{e e}^{2 g e n}$ is of the two generation form in the mixing angle $\theta_{12}$.

$$
P_{e e}^{2 g e n}=P_{e e}^{d a y}+\frac{\left(2 P_{e e}^{d a y}-1\right)\left(\sin ^{2} \theta_{12}-\left|A_{2 e}^{\oplus}\right|^{2}\right)}{\cos 2 \theta_{12}},
$$

where

$$
P_{e e}^{d a y}=0.5+\left[0.5-\Theta\left(E-E_{A}\right) X_{12}\right] \cos 2 \theta_{12}^{\odot} \cos 2 \theta_{12},
$$

with

$$
\tan 2 \theta_{12}^{\odot}=\frac{\Delta m_{21}^{2} \sin 2 \theta_{12}}{\Delta m_{21}^{2} \cos 2 \theta_{12}-A c_{13}^{2}}
$$

where $A$ denotes the matter potential,

$$
A=2 \sqrt{2} G_{F} n_{e}^{\odot} E
$$

here $n_{e}^{\odot}$ is the electron density in the sun, $E$ the neutrino energy, and $\Delta m_{21}^{2}$ $\left(=m_{2}^{2}-m_{1}^{2}\right)$ the mass squared difference in vacuum. The jump probability $X_{12}$ continues to be given by the two-generation expression and for this we use the analytic expression given in [27]. $E_{A}$ in the Heaviside function $\Theta$ gives the minimum $\nu_{e}$ energy that can encounter a resonance inside the sun and is given by

$$
E_{A}=\Delta m_{21}^{2} \cos 2 \theta_{12} /\left.2 \sqrt{2} G_{F} n_{e}\right|_{p r},
$$

gives the minimum $\nu_{e}$ energy that can encounter a resonance inside the sun, $\left.n_{e}\right|_{p r}$ being the electron density at the point of production. In the limit $\theta_{13}=$ 0 one recovers the two generation limit.

\subsection{The Probability for $\mathrm{CHOOZ}$}

The survival probability relevant for the $\mathrm{CHOOZ}$ experiment for the three generation case is

$$
\begin{aligned}
P_{e e}= & 1-c_{13}^{4}\left[\sin ^{2} 2 \theta_{12} \sin ^{2} \frac{\Delta m_{21}^{2} L}{4 E}\right]-\sin ^{2} 2 \theta_{13} \sin ^{2} \frac{\Delta m_{31}^{2} L}{4 E} \\
& +\sin ^{2} 2 \theta_{13} s_{12}^{2}\left[\sin ^{2} \frac{\Delta m_{31}^{2} L}{4 E}-\sin ^{2} \frac{\left(\Delta m_{31}^{2}-\Delta m_{21}^{2}\right) L}{4 E}\right]
\end{aligned}
$$

Since the average energy of the neutrinos in the CHOOZ experiment is $\sim 1$ $\mathrm{MeV}$ and the distance traveled by the neutrinos is of the order of $1 \mathrm{Km}$ the $\sin ^{2}\left(\frac{\Delta m_{21}^{2} L}{4 E}\right)$ term is important only for $\Delta m_{21}^{2} \gtrsim 3 \times 10^{-4} \mathrm{eV}^{2}$. The last term in the above expression is an interference term between both mass scales 28] and is absent if one uses the approximation $\Delta_{31}=\Delta_{32}$ and is often ignored. 


\section{The $\chi^{2}$ analysis}

The definition of $\chi_{\odot}^{2}$ used in our fits is

$$
\begin{aligned}
\chi_{\odot}^{2}= & \sum_{i, j=1,4}\left(R_{i}^{\mathrm{th}}-R_{i}^{\mathrm{exp}}\right)\left[\left(\sigma_{i j}^{\text {rates }}\right)^{2}\right]^{-1}\left(R_{j}^{\mathrm{th}}-R_{j}^{\mathrm{exp}}\right) \\
& +\sum_{i, j=1.38}\left(X_{n} S_{i}^{\mathrm{th}}-S_{i}^{\exp }\right)\left[\left(\sigma_{i j}^{\text {spm }}\right)^{2}\right]^{-1}\left(X_{n} S_{j}^{\mathrm{th}}-S_{j}^{\mathrm{exp}}\right)
\end{aligned}
$$

where $R_{i}^{\xi}$ ( $\xi=$ th or $\exp$ ) denote the total rate while $S_{i}^{\xi}$ denote the SK spectrum in the $\mathrm{i}^{\text {th }}$ bin. Both the experimental and theoretical values of the fitted quantities are normalised relative to the BPB00 [29] predictions. The experimental values for the total rates are the ones shown in Table 1, while the SK day-night spectra are taken from [2]. The error matrix $\left(\sigma^{\text {rates }}\right)^{2}$ contains the experimental errors, the theoretical errors (which includes error in the capture cross-sections and the astrophysical uncertainties in BPB00 predictions) along with their correlations. It is evaluated using the procedure of [30]. The error matrix for the spectrum $\left(\sigma^{s p m}\right)^{2}$ contains the correlated and uncorrelated errors as discussed in [31]. The details of the solar code used is described in [10, 32, 12, 14]. We vary the normalisation of the SK spectrum $X_{n}$ as a free parameter to avoid double counting with the SK data on total rate. Thus there are $(38-1)$ independent data points from the SK day-night spectrum along with the 4 total rates giving a total of 41 data points. For the analysis of only the solar data in the three-generation scheme, we have $(41-3)$ degrees of freedom (DOF). The best-fit values of parameters and the $\chi_{\min }^{2}$ are

- $\Delta m_{21}^{2}=4.7 \times 10^{-5} \mathrm{eV}^{2}, \tan ^{2} \theta_{12}=0.375, \tan ^{2} \theta_{13}=0.0, \chi_{\min }^{2}=33.42$

Hence the best-fit comes in the two-generation limit presented in 110, 12, 14].

We next incorporate the results from the CHOOZ reactor experiment [20]. The definition of $\chi_{\mathrm{CHOOZ}}^{2}$ is given by [33]

$$
\chi_{C H O O Z}^{2}=\sum_{j=1,15}\left(\frac{x_{j}-y_{j}}{\Delta x_{j}}\right)^{2}
$$

where $x_{j}$ are the experimental values, $y_{j}$ are the corresponding theoretical predictions, $\Delta x_{j}$ are the $1 \sigma$ errors in the experimental quantities and the sum is over 15 energy bins of data of the CHOOZ experiment [20]. The global $\chi^{2}$ for solar $+\mathrm{CHOOZ}$ analysis is defined as

$$
\chi_{\text {global }}^{2}=\chi_{\odot}^{2}+\chi_{C H O O Z}^{2}
$$

The total number of data points for combined solar and CHOOZ analysis is therefore $41+15=56$. The solar $+\mathrm{CHOOZ}$ analysis depends on $\Delta m_{21}^{2}, \Delta m_{31}^{2}$, $\theta_{12}$ and $\theta_{13}$. For unconstrained $\Delta m_{31}^{2}$, the $\chi_{\min }^{2}$ and the best-fit values are 
- $\Delta m_{21}^{2}=4.7 \times 10^{-5} \mathrm{eV}^{2}, \tan ^{2} \theta_{12}=0.374, \Delta m_{31}^{2}=1.35 \times 10^{-3} \mathrm{eV}^{2}$, $\tan ^{2} \theta_{13}=1.74 \times 10^{-3}, \chi_{\text {min }}^{2}=39.75$

However the atmospheric neutrino data imposes strong constraints on the allowed range of $\Delta m_{31}^{2}$. The combined analysis of the 1289 day atmospheric data and the $\mathrm{CHOOZ}$ data restricts allowed $\Delta m_{31}^{2}$ in the range $[1.5,6] \times 10^{-3}$ $\mathrm{eV}^{2}$ at $99 \%$ C.L. [21]. Thus the best-fit $\Delta m_{31}^{2}=1.35 \times 10^{-3}$ that we obtain from the solar $+\mathrm{CHOOZ}$ analysis falls outside the allowed range. If we restrict the range of $\Delta m_{31}^{2}$ from the combined analysis of the atmospheric $+\mathrm{CHOOZ}$ analysis [21] then the $\chi_{\min }^{2}$ and the best-fit parameters obtained from the combined solar $+\mathrm{CHOOZ}$ analysis are

$$
\begin{aligned}
& -\Delta m_{21}^{2}=4.7 \times 10^{-5} \mathrm{eV}^{2}, \tan ^{2} \theta_{12}=0.374, \Delta m_{31}^{2}=1.5 \times 10^{-3} \mathrm{eV}^{2}, \\
& \tan ^{2} \theta_{13}=1.46 \times 10^{-3}, \chi_{\text {min }}^{2}=39.75
\end{aligned}
$$

Thus the best-fit for the solar+CHOOZ analysis comes almost at the two generation limit, with the best-fit $\Delta m_{31}^{2}$ at the lower limit of the allowed range. For 52 DOF this solution is allowed at 89.33\%. The improvement in the goodness of fit (GOF) in comparison to the two flavour analysis presented in [10, 12, 14] is due to the inclusion of the CHOOZ data which gives a $\chi^{2} / \mathrm{DOF}$ of about $6 / 15$.

\section{Allowed areas in the three generation pa- rameter space}

\subsection{Constraints on the $\Delta \mathbf{m}_{31}^{2}-\tan ^{2} \theta_{13}$ plane}

For the chosen mass spectrum and mixing matrix the relevant survival probabilities for atmospheric neutrinos depend on the parameters $\theta_{23}, \theta_{13}$ and $\Delta \mathrm{m}_{32}^{2}\left(\simeq \Delta \mathrm{m}_{31}^{2}\right)$ [22] while the CHOOZ survival probability $\mathrm{P}_{\bar{e} \bar{e}}$ depends mainly on $\theta_{13}$ and $\Delta \mathrm{m}_{31}^{2}$ and very mildly on $\theta_{12}$ and $\Delta \mathrm{m}_{21}^{2}$. In fig. 1 we plot the allowed domains in the $\tan ^{2} \theta_{13}-\Delta m_{31}^{2}$ parameter space the from analysis of only the CHOOZ data keeping all other parameters free. We give this plot both with and without taking into account the interference term. The effect of the interference term is to lift the allowed ranges of $\Delta m_{31}^{2}$. The shaded area marked by arrows in this figure is the allowed range from a combined analysis of 1289 day atmospheric data and CHOOZ data taken from [21]. At 99\% C.L. the atmospheric $+\mathrm{CHOOZ}$ analysis allows $\tan ^{2} \theta_{13} \lesssim 0.08$ and $1.5 \times 10^{-3} \mathrm{eV}^{2}$ $<\Delta \mathrm{m}_{31}^{2}<6.0 \times 10^{-3} \mathrm{eV}^{2}$. It also becomes apparent from this figure that for $\tan ^{2} \theta_{13} \lesssim 0.03$, all values of $\Delta \mathrm{m}_{31}^{2}$ in the range $[1.5,6.0] \times 10^{-3} \mathrm{eV}^{2}$ are allowed at $99 \%$ C.L. where as for $0.03 \lesssim \tan ^{2} \theta_{13} \lesssim 0.075$, certain values of $\Delta m_{13}^{2}$ 
get excluded. A closer inspection of fig. 1 shows that around $\tan ^{2} \theta_{13} \sim 0.03$ a window in $\Delta m_{31}^{2}$ is disallowed whereas for higher values of $\tan ^{2} \theta_{13}$ certain regions of $\Delta m_{13}^{2}$ towards higher values of the interval $[1.5,6.0] \times 10^{-3} \mathrm{eV}^{2}$ get disallowed. The width of the disallowed range in $\Delta \mathrm{m}_{31}^{2}$ depend on $\tan ^{2} \theta_{13}$. Clearly the $\Delta \mathrm{m}_{31}^{2}$ is restricted more from the atmospheric data while the more stringent bound on $\tan ^{2} \theta_{13}$ comes from the CHOOZ results. It is also evident that the region in $\Delta m_{31}^{2}$ which is disallowed in the only $\mathrm{CHOOZ}$ contour once the interference effects are taken into account is also being disallowed by the combined atmospheric and $\mathrm{CHOOZ}$ analysis. In figs. $2 \mathrm{a}, 2 \mathrm{~b}$ and $2 \mathrm{c}$ we plot the $\chi_{\odot}^{2}, \chi_{C H O O Z}^{2}$, and $\chi_{\odot}^{2}+\chi_{C H O O Z}^{2}$ respectively against $\tan ^{2} \theta_{13}$, keeping $\theta_{12}$, $\Delta \mathrm{m}_{21}^{2}$ and $\Delta \mathrm{m}_{31}^{2}$ (in the range $[1.5,6.0] \times 10^{-3}$

$\mathrm{eV}^{2}$ ) free. It is clear from the three figures that the most stringent bound on $\tan ^{2} \theta_{13}(<0.065$ at $99 \%$ C.L. $)$ comes from the combined solar and CHOOZ analysis. The pre-SNO bound on $\tan ^{2} \theta_{13}$ that we get from the combined solar+CHOOZ analysis is $\tan ^{2} \theta_{13} \lesssim 0.075$. Thus SNO is seen to tighten the constraint on the $\theta_{13}$ mixing angle such that the most stringent upper limit on $\theta_{13}$ is obtained from the solar plus $\mathrm{CHOOZ}$ analysis.

\subsection{Probing the $\Delta \mathbf{m}_{21}^{2}-\tan ^{2} \theta_{12}$ parameter space.}

We now attempt to explore the 1-2 parameter space from a combined solar $+\mathrm{CHOOZ}$ analysis, in the light of new results from SNO. The parameters $\theta_{12}$ and $\Delta \mathrm{m}_{21}^{2}$ are mainly constrained from the solar data. We present in fig. 3 the allowed areas in the 1-2 plane at 90\%, 95\%, 99\% and 99.73\% confidence levels for different sets of combination of $\Delta m_{31}^{2}$ and $\tan ^{2} \theta_{13}$, lying within their respective allowed range from atmospheric $+\mathrm{CHOOZ}$ and solar $+\mathrm{CHOOZ}$ analysis. The CHOOZ data limits the upper allowed range of $\Delta m_{12}^{2}$ in the LMA region to $3 \times 10^{-4} \mathrm{eV}^{2}$. In the three flavor scenario also there is no room for SMA MSW solution at the $3 \sigma$ level $(99.73 \%$ C.L) l. We see from fig. 3 that the allowed regions reduce in size as we increase $\tan ^{2} \theta_{13}$ for a fixed $\Delta m_{31}^{2}$. At the upper limit of the allowed range of $\Delta \mathrm{m}_{31}^{2}$ the LOW solution gets completely disallowed beyond $\tan ^{2} \theta_{13} \sim 0.02$ while the LMA solution gets disallowed beyond $\tan ^{2} \theta_{13} \sim 0.03$. At the lower limit of of $\Delta \mathrm{m}_{31}^{2}$ the LMA solution is found to disappear at $99 \%$ C.L. beyond $\tan ^{2} \theta_{13} \sim 0.065$, which is the upper bound of $\tan ^{2} \theta_{13}$ at $99 \%$ C.L., obtained from solar+CHOOZ analysis. On the other hand for any given $\tan ^{2} \theta_{13}$ the least allowed area in $\tan ^{2} \theta_{12}-\Delta \mathrm{m}_{21}^{2}$ parameter

\footnotetext{
${ }^{6}$ We find that for values of $\tan ^{2} \theta_{13}>0.25$, one gets allowed areas in the SMA region at $3 \sigma$ level even after including the SNO data. Beyond this value of $\tan ^{2} \theta_{13}$ the allowed area in the SMA region increases and finally for larger values of $\tan ^{2} \theta_{13}$ the SMA and LMA regions merge with each other. However these large values of $\tan ^{2} \theta_{13}$ lie outside the range allowed by CHOOZ.
} 
space occurs at $\Delta \mathrm{m}_{13}^{2} \sim 4.0 \times 10^{-3} \mathrm{eV}^{2}$, whereas above and below this value larger regions of parameter space are allowed. To illustrate this in fig. 4 we plot the $\chi_{\odot}^{2}+\chi_{C H O O Z}^{2}$ vs. $\Delta m_{31}^{2}$ for fixed $\tan ^{2} \theta_{13}$ allowing the other parameters to vary freely. The highest value of $\chi^{2}$ is seen to come for $\Delta m_{31}^{2}=0.004$ $\mathrm{eV}^{2}$ explaining the least allowed area at this value. The figure also illustrates the occurrence of a disallowed window in $\Delta m_{31}^{2}$ around $\tan ^{2} \theta_{13} \sim 0.03$, as discussed earlier. Since the solar probabilities are independent of $\Delta m_{13}^{2}$ it is clear that the CHOOZ data is responsible for this feature. We have plotted these figures taking the interference term in the $\mathrm{CHOOZ}$ probability into account. However we have explicitly checked that the interfernce term in the CHOOZ probability does not have any impact on the allowed area in the $\Delta m_{21}^{2}-\tan ^{2} \theta_{12}$ plane. There are two reasons for this. The interference term comes multiplied with $s_{13}^{2}$ which is confined to very small values. Also the contours that we have plotted are for values of $\Delta m_{31}^{2}>1.5 \times 10^{-3} \mathrm{eV}^{2}$ as allowed by the combined atmospheric and CHOOZ analysis. As is seen from fig. 1 in this region the interference term does not have any significant effect.

\section{Summary, Conclusions and Discussions}

We have performed a three-generation analysis of the solar neutrino and $\mathrm{CHOOZ}$ data including the recent SNO CC results. The mass spectrum considered is one where $\Delta m_{21}^{2}=\Delta m_{\odot}^{2}$ and $\Delta m_{31}^{2} \approx \Delta m_{32}^{2}=\Delta m_{\text {atm }}^{2}=\Delta_{C H O O Z}$. The other parameters are the three mixing angles $\theta_{13}, \theta_{12}$ and $\theta_{23}$. For the combined solar and CHOOZ analysis the probabilities are independent of $\theta_{23}$. The solar neutrino probabilities depend on $\Delta m_{21}^{2}, \theta_{12}$ and $\theta_{13}$. The CHOOZ probability depends mainly on $\Delta m_{31}^{2}$ and $\theta_{13}$ whereas for $\Delta m_{12}^{2} \gtrsim 3 \times 10^{-4} \mathrm{eV}^{2}$ it depends also on $\Delta m_{12}^{2}$ and $\theta_{12}$. The most stringent constraint on the parameter $\Delta m_{31}^{2}$ comes from the atmospheric neutrino data. For this we use the updated values from [22, 21]. The combined atmospheric $+\mathrm{CHOOZ}$ analysis gives $\tan ^{2} \theta_{13} \lesssim 0.075$ [21, 22]. We keep $\Delta m_{31}^{2}$ in the range allowed by the atmospheric neutrino data and determine the allowed values of $\theta_{13}$ from a combined analysis of solar and $\mathrm{CHOOZ}$ data. The inclusion of the SNO results puts a more restrictive bound on $\theta_{13}-\tan ^{2} \theta_{13}<0.065$. The best-fit comes in the LMA region of the $\Delta m_{21}^{2}-\tan ^{2} \theta_{12}$ plane with $\tan ^{2} \theta_{13}=0.0$ i.e. at the two generation limit. We present the allowed region in the $\Delta m_{21}^{2}-\tan ^{2} \theta_{12}$ parameter space for various values of $\tan ^{2} \theta_{13}$ and $\Delta m_{31}^{2}$ belonging to their respective allowed ranges and determine the changes in the two-generation allowed region due to the presence of the mixing with the third generation. Since very low values of $\theta_{13}$ are allowed from combined solar and $\mathrm{CHOOZ}$ analysis there is not much change in the two generation allowed regions. No allowed area is obtained in the SMA region at $3 \sigma$ if one restricts $\tan ^{2} \theta_{13}$ to be $<0.065$, as 
allowed by combined solar and CHOOZ analysis.

The combination of solar, atmospheric and CHOOZ data allows to fix the elements of the neutrino mixing matrix. The $U_{e 3}$ element is narrowed down to a small range $\lesssim 0.255$ from the solar $+\mathrm{CHOOZ}$ analysis including SNO. The $\theta_{23}$ mixing angle is $\approx \pi / 4$ from atmospheric data [22, 21]. This determines the mixing matrix elements $U_{\mu 3}$ and $U_{\tau 3}$. The $\theta_{12}$ mixing angle is limited by the solar data and the tilt is towards large $\tan ^{2} \theta_{12}$. The mixing matrix at the best-fit value of solar $+\mathrm{CHOOZ}$ analysis is

$$
U \simeq\left(\begin{array}{ccc}
2 \sqrt{\frac{2}{11}} & \sqrt{\frac{3}{11}} & 0 \\
-\sqrt{\frac{3}{22}} & \frac{2}{\sqrt{11}} & \frac{1}{\sqrt{2}} \\
\sqrt{\frac{3}{22}} & -\frac{2}{\sqrt{11}} & \frac{1}{\sqrt{2}}
\end{array}\right)
$$

Thus the best-fit mixing matrix is one where the neutrino pair with larger mass splitting is maximally mixed whereas the pair with splitting in the solar neutrino range has large but not maximal mixing. It is a challenging task from the point of view of model building to construct such scenarios ๆ.

From the perspective of model building an attractive possibility is one where both pairs are maximally mixed [35]. Our two generation analysis of the solar data showed that for the LMA MSW region maximal mixing is not allowed at $99.73 \%$ C.L. though it is allowed for the LOW [10, 12] solution 8. However fig. 4 of this paper shows that three generation analysis allows $\tan ^{2} \theta_{12}=1.0$ with $\Delta m_{21}^{2}$ in the LMA region at $99.73 \%$ C.L. for $\Delta m_{31}^{2}$ in its lower allowed range $\sim 1.5 \times 10^{-3} \mathrm{eV}^{2}$ and for $\tan ^{2} \theta_{13} \sim 0.02$. As $\Delta m_{31}^{2}$ increases $\theta_{12}=\pi / 4$ in the LMA region no longer remains allowed even at $99.73 \%$ level though it remains allowed in the LOW-QVO region. Further narrowing down of the $\Delta m_{12}^{2}-\tan ^{2} \theta_{12}$ parameter space is expected to come from experiments like KamLand and Borexino which will be able to distinguish between the LMA and LOW regions.

S.G. wishes to acknowledge the kind hospitality extended to her by the theory group of Physical Research Laboratory.

Note added: After submission of our revised manuscript a preprint [36] appeared which finds constraints on $\left|U_{e 3}\right|^{2}$ from a similar three generation analysis of the CHOOZ data.

\section{References}

\footnotetext{
${ }^{7}$ For a recent study see 34 .

${ }^{8}$ See however [14].
} 
[1] SNO Collaboration: Q.R. Ahmad et al.., nucl-ex/0106015.

[2] Y. Fukuda et al.(The Super-Kamiokande collaboration), Phys. Rev. Lett. 81, 1158 (1998); erratum 81, 4279 (1998);

[3] B.T. Cleveland et al.Astrophys. J 496, 505 (1998).

[4] Y. Fukuda et al.., (The Kamiokande collaboration), Phys. Rev. Lett. 77, 1683 (1996).

[5] J.N. Abdurashitov et al., (The SAGE collaboration), Phys. Rev. Lett. 77, 4708 (1996); Phys. Rev. C 60, 055801 (1999); W. Hampel et al., (The Gallex collaboration), Phys. Lett. B388, 384 (1996); Phys. Lett. B447, 127 (1999); Talk presented in Neutrino 2000 held at Sudbury, Canada (T.A. Kirsten for The Gallex collaboration), Nucl. Phys. B Proc. Suppl. 77, 26 (2000); M. Altmann et al., (The GNO collaboration),Phys. Lett. B492,16 (2000); Talk presented in Neutrino 2000 held at Sudbury, Canada ( E. Belloti for the GNO Collaboration) Nucl. Phys. B Proc. Suppl. 9144 (2001).

[6] V. Barger, D. Marfatia and K. Whisnant, hep-ph/0106207.

[7] G.L. Fogli, E. Lisi, D. Montanino and A. Palazzo, hep-ph/0106247.

[8] C. Giunti, hep-ph/0107310.

[9] J.N. Bahcall, M.C. Gonzalez-Garcia and C. Pana-Garay, hep-ph/0106258.

[10] A. Bandyopadhyay, S. Choubey, S. Goswami and K. Kar, Phys. Lett. B519, 83 (2001).

[11] P. Creminelli, G. Signorelli, A. Strumia, hep-ph/0102234, updated version.

[12] S. Choubey, S. Goswami, K. Kar, H.M. Antia and S.M. Chitre, Phys. Rev. D64, (in press), hep-ph/0106168.

[13] P.I. Krastev and A.Yu. Smirnov, hep-ph/0108177.

[14] S. Choubey, S. Goswami and D.P. Roy, hep-ph/0109017.

[15] M.V. Garzelli and C. Giunti, hep-ph/0108191.

[16] Y. Fukuda et al., The Super-Kamiokande Collaboration, Phys. Lett. B433, 9 (1998); Phys. Lett. B436, 33 (1998); Phys. Rev. Lett. 81, 1562 (1998). S. Fukuda et al., The Super-Kamiokande Collaboration, hepex/0009001. 
[17] C. Athanassopoulos et al., Phys. Rev. Lett. 75, 2650 (1995); C. Athanassopoulos et al., Phys. Rev. Lett. 81, 1774 (1998); Talk presented by the LSND Collaboration in Neutrino 2000, Sudbury, Canada, 2000.

[18] J.J. Gomez-Cadenas and M.C. Gonzalez-Garcia, Z. Phys. C71, 443 (1996); N. Okada and O. Yasuda, Int. J. Mod. Phys. A12, 3669 (1997); S. Goswami, Phys. Rev. D55, 2931 (1997); S. M. Bilenky, C. Giunti, and W. Grimus, Phys. Rev. D57, 1920 (1998) and D58, 033001 (1998); V. Barger, S. Pakvasa, T. J. Weiler, and K. Whisnant, Phys. Rev. D58, 093016 (1998).

[19] M.C. Gonzalez-Garcia, M. Maltoni and C. Pena-Garay, hep-ph/0108073 and references therein K.S. Babu and R.N. Mohapatra, hep-ph/0110243; S. Goswami and A.Joshipura, hep-ph/0110272.

[20] M. Appolonio et al., Phys. Lett. B466, 415 (1999); Phys. Lett. B420, 397 (1998).

[21] G.L.Fogli, E.Lisi, A.Montamino and A.Palazzo, hep-ph/0104221.

[22] M.C. Gonzalez-Garcia, M.Maltoni, C. Pena-Garay and J.W.F.Valle, Phys. Rev. D63, 033005, (2001).

[23] R. Barbieri et al., JHEP, 9812, 017 (1998); V. Barger and K. Whisnant, Phys. Rev. D59, 093007, (1999).

[24] We have generalised the approach given in G.L. Fogli, E.Lisi, D. Montanino and A. Palazzo, Phys. Rev. D62, 113004, (2000) for three flavours.

[25] S.T. Petcov, Phys. Lett. B434, 321 (1998); M. Narayan, G. Rajasekharan and R. Sinha, Mod. Phys. Lett. A13, 1915 (1998).

[26] S.T. Petcov, Phys. Lett. B214 (1988) 139; Phys. Lett. B406, 355 (1997), S.T. Petcov and J. Rich PL B214, 137 , (1989).

[27] S.T. Petcov, Phys. Lett. B200, 373 (1988).

[28] S.T. Petcov and M. Piai, hep-ph/0112074.

[29] J.N. Bahcall, S. Basu, M.P. Pinsonneault, Astrophys. J. 555, 990 (2001).

[30] G.L. Fogli, E. Lisi, Astropart. Phys. 3, 185 (1995).

[31] M.C. Gonzalez-Garcia, P.C. de Holanda, C. Peña-Garay, and J.W.F. Valle, Nucl. Phys. B573, 3 (2000). 
[32] S. Goswami, D. Majumdar, A. Raychaudhuri, Phys. Rev. D63, 013003 (2001); hep-ph/9909453; A. Bandyopadhyay, S. Choubey and S. Goswami, Phys. Rev. D63, 113019 (2001); S. Choubey, S. Goswami, N. Gupta and D.P. Roy, Phys. Rev. D64, 053002 (2001).

[33] R. Foot, R.R. Volkas and O. Yasuda, Phys. Rev. D58 013006, (1998);

S. Choubey, S. Goswami and K. Kar, Astropart. Phys. (in press), hep$\mathrm{ph} / 0004100$.

[34] W. Grimus and L. Lavoura, hep-ph/0110041.

[35] See for example V. Barger et al., Phys. Lett. B437, 107 (1998).

[36] S. M. Bilenky, D. Nicclo and S. T. Petcov, hep-ph/0112216. 
Table 1: The ratio of the observed solar neutrino rates to the corresponding BPB00 SSM predictions.

\begin{tabular}{ccc}
\hline experiment & $\frac{\text { observed }}{B P B 00}$ & composition \\
\hline $\mathrm{Cl}$ & $0.335 \pm 0.029$ & $B(75 \%), B e(15 \%)$ \\
$\mathrm{Ga}$ & $0.584 \pm 0.039$ & $p p(55 \%), B e(25 \%), B(10 \%)$ \\
$\mathrm{SK}$ & $0.459 \pm 0.017$ & $B(100 \%)$ \\
$\mathrm{SNO}(\mathrm{CC})$ & $0.347 \pm 0.027$ & $B(100 \%)$ \\
\hline
\end{tabular}




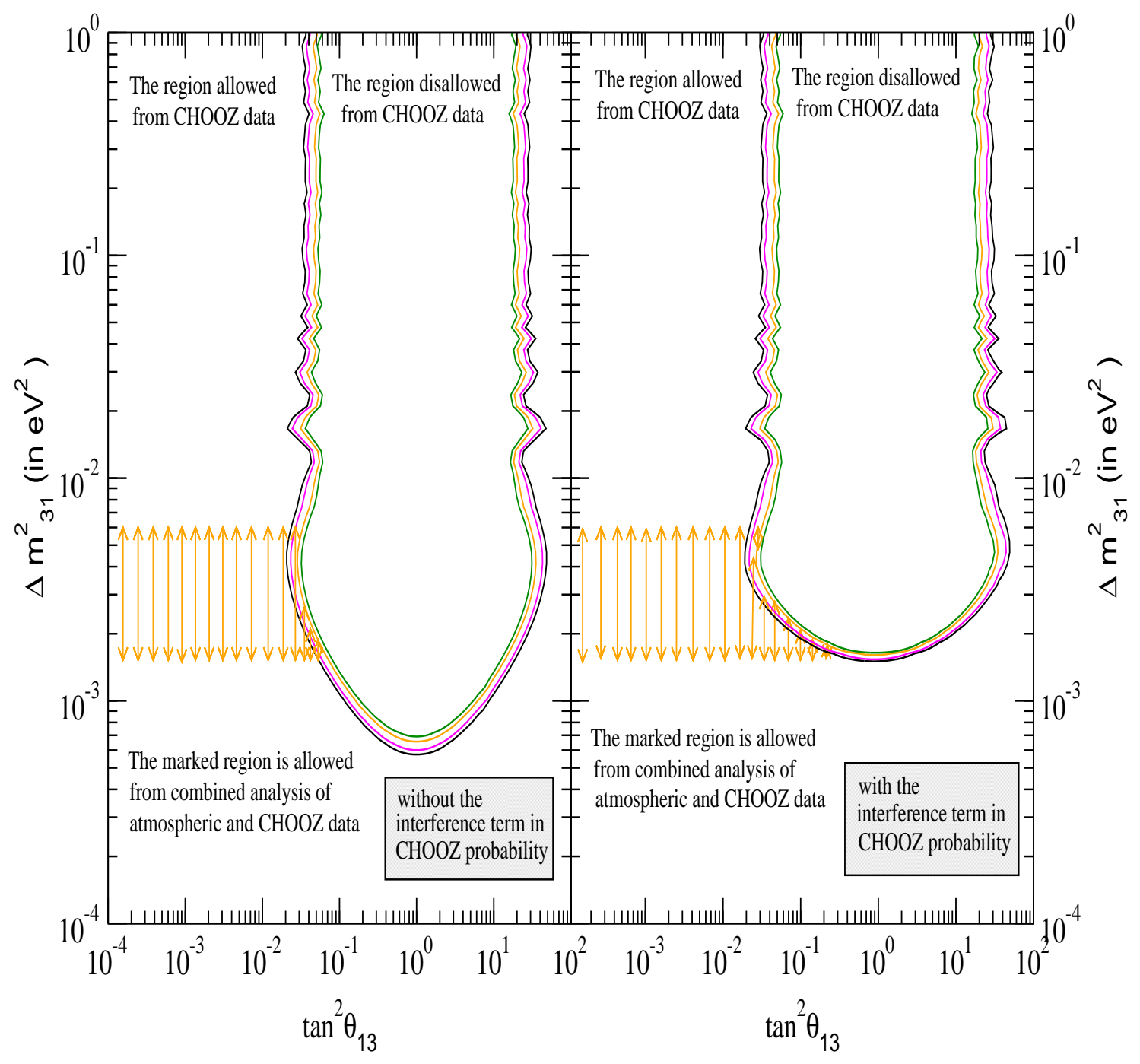

Figure 1: The allowed areas in $\left(\tan ^{2} \theta_{13}-\Delta m_{31}^{2}\right)$ plane from atmospheric and CHOOZ data. 

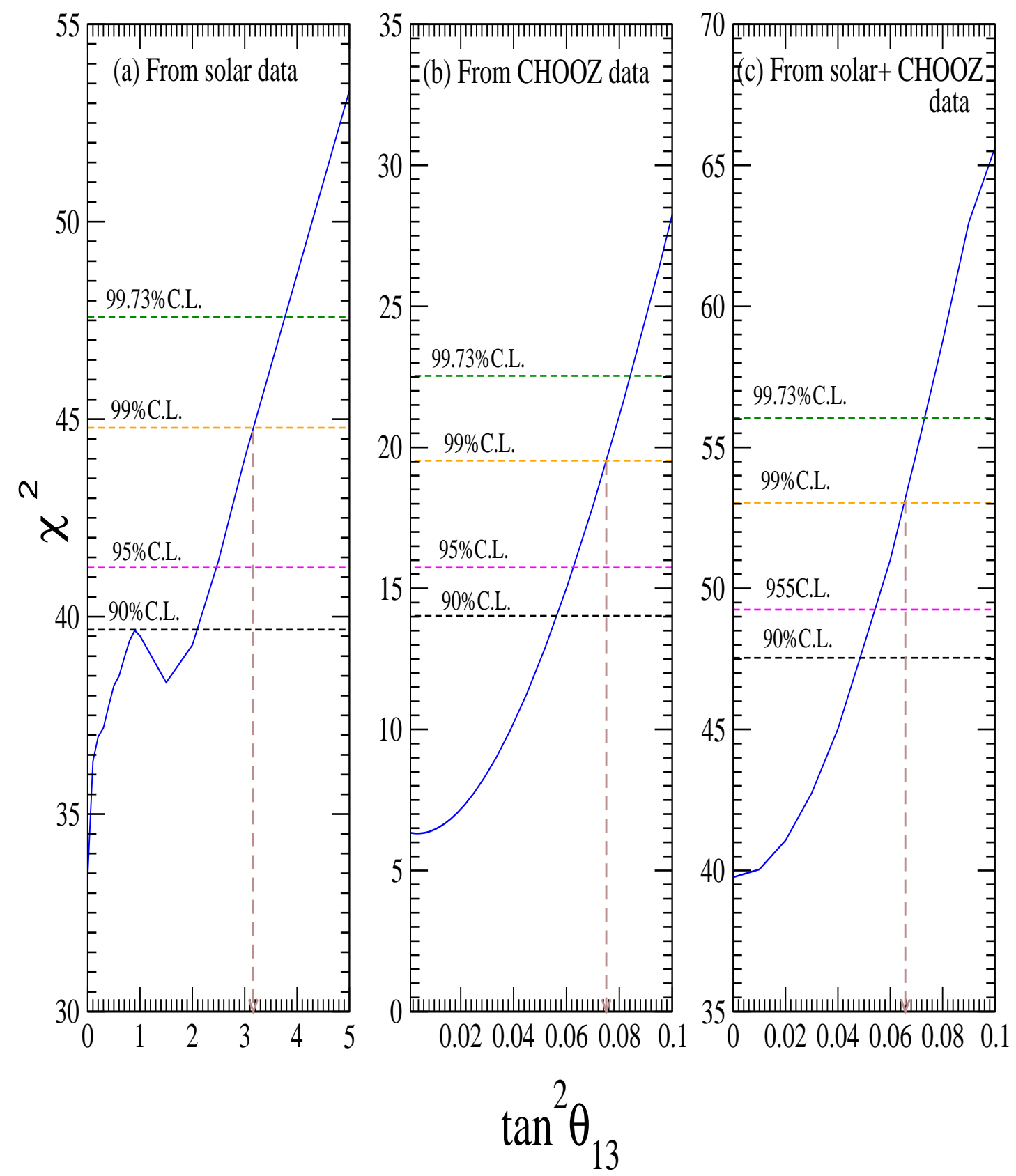

Figure 2: The plot of $\chi^{2}$ vs $\tan ^{2} \theta_{13}$ from (a) solar (b) CHOOZ and (c) solar+CHOOZ data. 


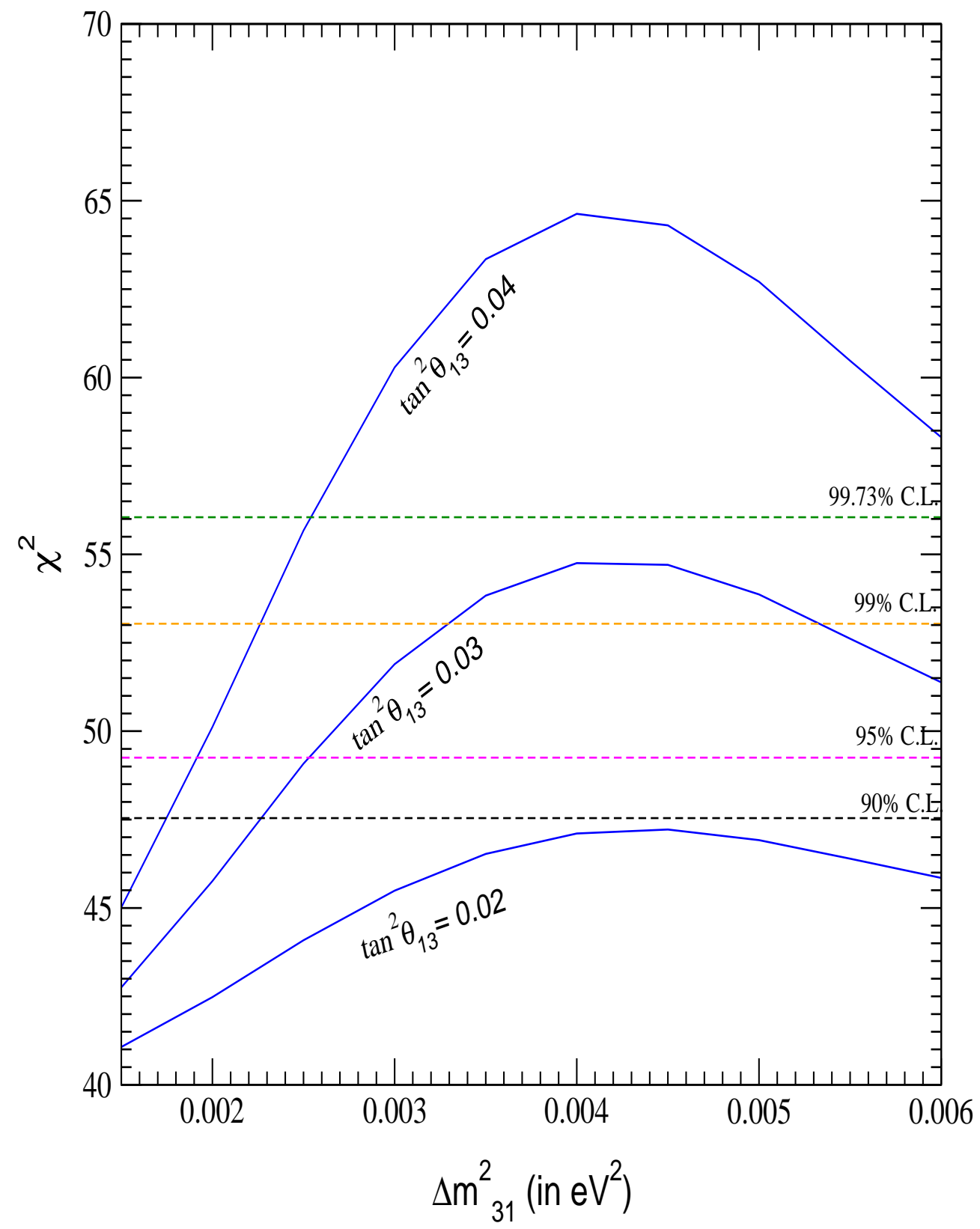

Figure 3: The plot of $\chi^{2}$ vs $\Delta \mathrm{m}_{31}^{2}$ from solar $+\mathrm{CHOOZ}$ data. 


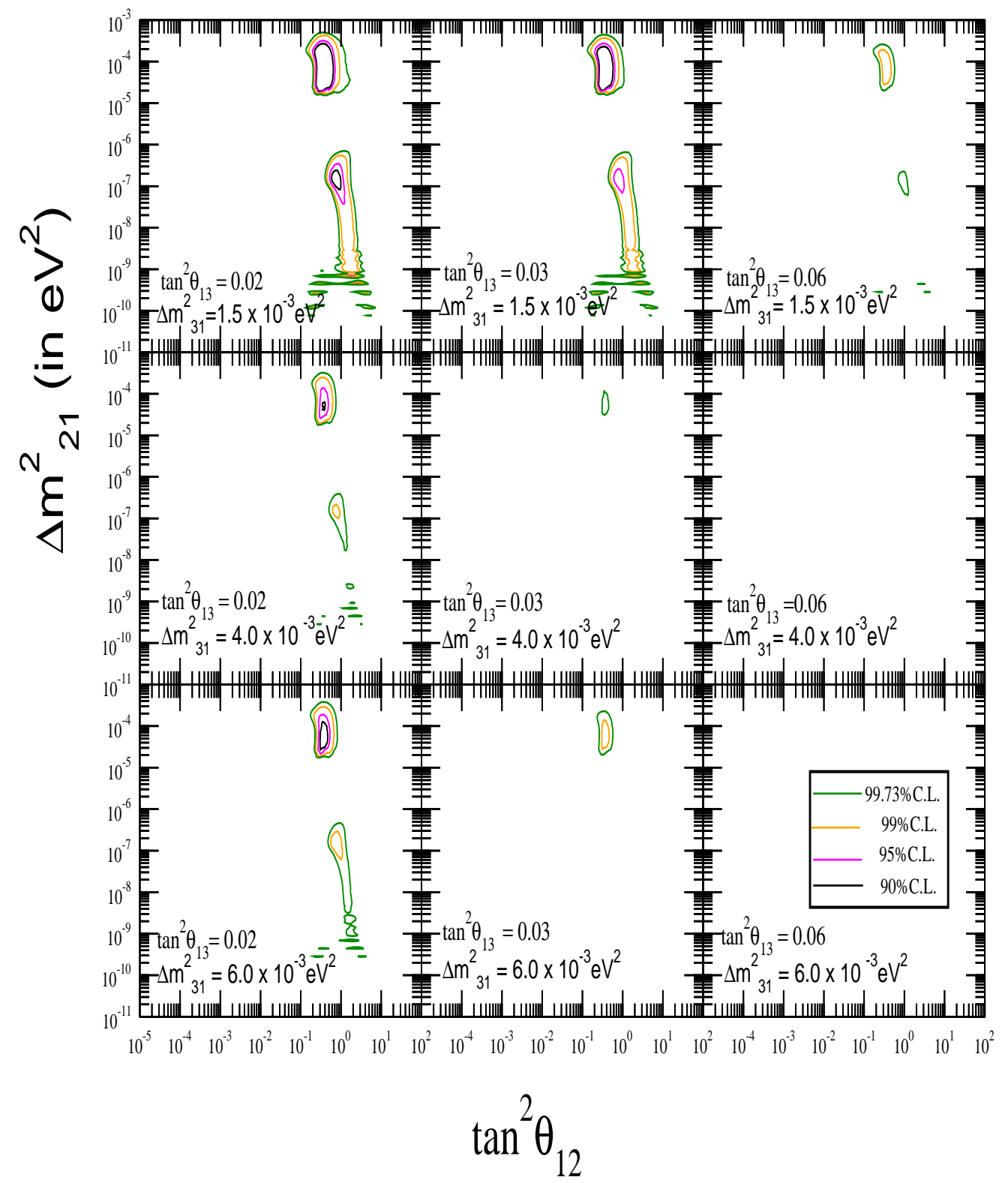

Figure 4: The allowed areas in $\left(\tan ^{2} \theta_{12}-\Delta \mathrm{m}_{21}^{2}\right)$ plane from solar+CHOOZ analysis. 